\title{
Donor directed lobar lung transplantation
}

\author{
Daniel Rinewalt ${ }^{1}$, Michael Wong ${ }^{2}$, Sylvia M. Cruz ${ }^{2}$, Francis Fynn-Thompson ${ }^{3}$, Steve Singh ${ }^{4}$ \\ ${ }^{1}$ Division of Cardiac Surgery, Brigham and Women's Hospital, Boston, MA, USA; ${ }^{2}$ Department of Cardiothoracic Surgery, Stanford University, \\ Palo Alto, California, USA; ${ }^{3}$ Department of Cardiac Surgery, Boston Children's Hospital, Boston, MA, USA; ${ }^{4}$ Trillium Health Partners, Mississauga, \\ Ontario, Canada \\ Correspondence to: Daniel Rinewalt, MD. Division of Cardiac Surgery, Brigham and Women's Hospital, 75 Francis Street, Boston, MA 02115, USA. \\ Email: drinewalt@bwh.harvard.edu.
}

Submitted Sep 02, 2019. Accepted for publication Oct 30, 2019.

doi: $10.21037 /$ acs.2019.11.02

View this article at: http://dx.doi.org/10.21037/acs.2019.11.02

Lung transplantation remains the gold standard surgical treatment for patients with end-stage lung failure from a variety of causes; however for the foreseeable future, will continue to be a limited modality, secondary to a critical shortage of suitable donor organs (1). Living lobar lung transplantation, a technique pioneered by Dr. Vaughn Starnes at the University of Southern California (USC), involves locating two donors per recipient and harvesting a lobe from each for implantation. This strategy reached its peak popularity in the late 1990's in the United States with several centers, notably USC and Washington University in St. Louis, reporting favorable outcomes for donor-directed lobar lung transplantation (2). The implementation of the lung allocation score (LAS) (3) in 2005 catalyzed a drastic change in the way organs are matched to recipients, moving away from the previous time-based system on the transplant waiting list and towards a system that prioritized critically ill patients (4). This allocation strategy has led to a decrease in waitlist mortality and an increase in the overall number of transplants performed in the United States (4). Additionally, along with improved medical management options for certain lung diseases, donor-directed lobar transplantation has steadily decreased in frequency.

Overall, outcomes from living lobar lung transplantation have been favorable and consistently comparable to or even superior to survival from brain-dead donors (5). Lack of widespread adoption of this technique by other centers remains primarily related to the potential risk to donors and the ethical dilemmas related to their involvement. The operation itself is technically challenging, in that unlike a typical lobectomy, the portion of lung must be harvested in such a way as to preserve adequate cuffs of bronchus, vein and artery for reimplantation. In the Renal and Lung Living Donors Evaluation Study (RELIVE), serious complications (i.e., defined as those requiring significant treatment, potentially life-threatening, or leading to prolonged hospitalization) to lung donors were reported in $18 \%$ of donors, with $2 \%$ of these undergoing reoperation (2). Specific donor complications have ranged from pleural effusions to bronchial stump fistulae, strictures, atrial fibrillation and damage to the phrenic nerve (6). Importantly, no donor mortality has been reported in the literature. Certainly, the donor evaluation remains rigorous from a physiologic standpoint; however, it is difficult to determine the psychological impact on these volunteers. Chen et al. reported the results of a survey study in 2013 showing a decrease in donor quality of life after surgery, especially in cases where the recipient experienced a poor outcome (7). The family dynamics in such a situation as living lobar transplantation are challenging and while physicians have gone to great lengths to maintain the highest ethical standards, there are many situations where one can imagine significant difficulty. In the most straightforward sense, any living donor is subject to serious harm or mortality without any physiologic or survival benefit to themselves. Beyond the obvious risks to donors, physicians have been careful to ensure that no coercion exists in the donation process; however, one can imagine that any relative would feel pressured to donate if many others readily volunteered. The recipients themselves likely experience a range of emotions putting family members or friends in such a position, making these procedures arguably some of the most psychosocially complex in all of modern medicine. 
In the modern era, the use of the LAS in the United States as well as continued success with extended criteria donors, donation after cardiac death and ex-vivo lung perfusion, continues to increase the donor pool, therefore making it more difficult to justify the potential risks of donor-directed lobar lung transplantation. There remain specific situations where this technique is favorable and can be performed successfully at specialized centers. Generally, any patient with a significantly high risk of mortality on the waitlist combined with a low probability of receiving a suitable donor lung in a reasonable amount of time, can be referred to a high-volume center to be considered for this strategy. Patients of short stature or children typically have a much longer waitlist time with increased mortality and can certainly have an excellent outcome with implanted adult-sized lobes. Those who are highly sensitized from previous procedures may never obtain an acceptable crossmatch from the cadaveric donor pool at large, although these patients may benefit from a favorable immunologic profile provided by a close relative. Some countries, notably Japan, have less available donor organs overall, due to complex social reasons related to the legal and ethical concerns regarding pronouncement of brain death (5). Living lobar lung transplantation technique has thrived at several Japanese centers, such that they account for the majority of these procedures in the modern era (5).

In conclusion, donor-directed lobar lung transplantation has decreased in frequency over the past 25 years due to improvements in the lung allocation system in the United States. There remain several indications where this strategy can successfully be performed at specialized centers with excellent outcomes.

Cite this article as: Rinewalt D, Wong M, Cruz SM, Fynn-Thompson F, Singh S. Donor directed lobar lung transplantation. Ann Cardiothorac Surg 2020;9(1):56-57. doi: 10.21037/acs.2019.11.02

\section{Acknowledgments}

None.

\section{Footnote}

Conflicts of Interest: The authors have no conflicts of interest to declare.

\section{References}

1. Organ Procurement and Transplantation Network. (U.S.) [Online] (2019). Transplants in the U.S. National Data. Available online: https://optn.transplant.hrsa.gov/data/ view-data-reports/national-data/

2. Yusen RD, Hong BA, Messersmith EE, et al. Morbidity and mortality of live lung donation: results from the RELIVE study. Am J Transplant 2014;14:1846-52.

3. Organ Procurement and Transplantation Network (U.S.) [Online] (2019). LAS Calculator. Available online: https:// optn.transplant.hrsa.gov/resources/allocation-calculators/ las-calculator/

4. Egan TM, Edwards LB. Effect of the lung allocation score on lung transplantation in the United States. J Heart Lung Transplant 2016;35:433-9.

5. Date H, Aoe M, Sano Y, et al. Improved survival after living-donor lobar lung transplantation. J Thorac Cardiovasc Surg 2004;128:933-40.

6. Battafarano RJ, Anderson RC, Meyers BF, et al. Perioperative complications after living donor lobectomy. J Thorac Cardiovasc Surg 2000;120:909-15.

7. Chen F, Oga T, Sakai H, et al. A prospective study analyzing one-year multidimensional outcomes in living lung transplant donors. Am J Transplant 2013;13:3003-9. 